\title{
Fabric evolution and accessible geometrical states in granular materials
}

\author{
F. Radjai · J.-Y. Delenne · E. Azéma · S. Roux
}

Received: 26 September 2011

(C) Springer-Verlag 2012

\begin{abstract}
We analyze the geometrical states of granular materials by means of a fabric tensor involving the coordination number and fabric anisotropy as the lowest-order descriptors of the contact network. In particular, we show that the fabric states in this representation are constrained by steric exclusions and the condition of mechanical equilibrium required in the quasi-static limit. A simple model, supported by numerical data, allows us to characterize the range of accessible fabric states and the joint evolution of fabric parameters. The critical state in this framework appears as a jammed state in the sense of a saturation of contact gain and loss along the principal strain-rate directions.
\end{abstract}

Keywords Plastic behavior - Granular fabric . Coordination number $\cdot$ Anisotropy $\cdot$ Mohr circle

\section{Introduction}

The plasticity of granular materials is governed by frictional contact interactions among a large number of rigid-body degrees of freedom. The particle velocities are constrained by steric exclusions and, in the absence of thermal randomness, they scale with the driving strain. In a similar vein, the material is devoid of intrinsic stress scale in the limit of rigid particles (compared to the applied confining stress), and thus all internal stresses scale with the confining stress while sat-

F. Radjai ( $\varangle)$ · J.-Y. Delenne · E. Azéma

LMGC, CNRS-Université Montpellier 2, Place Eugène Bataillon, 34095 Montpellier, France

e-mail: franck.radjai@univ-montp2.fr

S. Roux

LMT-Cachan, ENS de Cachan/CNRS/UPMC/PRES UniverSud Paris, 61 Av. Président Wilson, 94235 Cachan Cedex, France isfying at the same time the condition of static equilibrium of each particle $[6,4]$. These features lead to a perfectly plastic behavior in which the instantaneous flow rule is defined by a strain ratio expressed as a dilatancy angle and the yield surface by a stress ratio expressed as an effective friction angle [9]. These angles are unequal (non-associated property) due to steric exclusions and, more importantly, they are strongly dependent on the current state of the material, which is fully encoded in the geometry of the granular assembly and evolves with strain (hardening property).

The local geometrical arrangement is nontrivial regarding its generic disorder both in particle positions and in the forcebearing contact network, and its evolution involves local nonaffine velocities induced by steric exclusions [3]. However, a coarse-grained representation of this microstructure is a key information for any continuum model of granular plasticity based on physical internal variables. This issue is still far from being settled even when approaching the problem under simplified assumptions such as spherical particles subjected to homogeneous shearing. The heart of the problem is whether one may work with the lowest-order descriptors of the microstructure-possibly at the price of accounting in a less strict sense for the local constraints. In particular, such state variables are expected to provide a minimal description of the reference states such as the critical state and the spectrum of isotropic states with its range of packing fractions, as well as the transients between those reference states.

In this paper, the contact network is portrayed by its lowest-order representation as a tensor combining the coordination number and fabric anisotropy. Its evolution in relation with the velocity field is studied by means of numerical simulations for a few loading paths. The idea is to characterize the range of accessible fabric states constrained by steric effects and force balance condition. A simple model is presented that provides a geometrical interpretation of the critical state. 


\section{Tensorial representation of the contact network}

The granular microstructure may be described by the contact network, which is directly linked with the plastic behavior as it governs both stress transmission and volume change by shear flow. This granular fabric is disordered in (1) contact positions, (2) contact orientations, as described by the normals to the contact planes or the orientations $\boldsymbol{n}$ of the branch vectors $\ell=\ell \boldsymbol{n}$ joining the centers of contact neighbors, and (3) branch-vector lengths $\ell$; see Fig. 2a.

Assuming a statistically homogeneous network, as shown in Fig. 1, the fabric is thus represented by the set $\left\{\ell^{\alpha}\right\}$ of branch vectors and characterized by the probability density $P_{\ell n}(\ell) \equiv P_{\ell n}(\ell, \boldsymbol{n})$. For non-spherical particles, this function may be replaced by the richer probability density function of contact vectors, i.e. the vectors joining the particle centers to the contact points; Fig. 2a. Note that since the branch vectors $\ell^{\alpha}$ have no intrinsic polarity, the function $P_{\ell n}(\ell)$ is $\pi$-periodic.

The function $P_{\ell n}(\ell)$ is obviously insufficient for the description of individual particle environments (one particle with its contact neighbors), which involve multicontact distributions constrained by angular hinderances $\delta \theta$ as illustrated in Fig. 2b [8]. Although such a rich representation would be required in order to account for the local geometrical constraints together with higher order statistical information on three (and more) grain locations and orientations, the idea of the proposed approach is to include a minimal geometrical description and to connect it to the mechanical behavior. A scalar variable that provides the lowest-order description of the particle environments is the average number of contact neighbors $z$ (the coordination number), which is a complementary information to $P_{\ell n}$. Additional local constraints will have to be reintroduced at a later stage from coarse-grained or average properties.

The reduced distribution $P_{\ell}(\ell)=\int_{\Omega} P_{\ell n}(\ell) d n$ of branchvector lengths, where $\Omega$ is the space of orientations, reflects the particle size distribution. The correlation between the orientations $\boldsymbol{n}$ and lengths $\ell$ controls the mean branch-vector length $\langle\ell\rangle(\boldsymbol{n})=\int_{0}^{\infty} \ell P_{\ell n}(\ell, \boldsymbol{n}) d \ell / \int_{0}^{\infty} P_{\ell n}(\ell, \boldsymbol{n}) d \ell$ as a function of $\boldsymbol{n}$. Our simulations show that this function in a moderately polydisperse packing is nearly isotropic even after extended shear [1]. We focus here on weakly polydis-

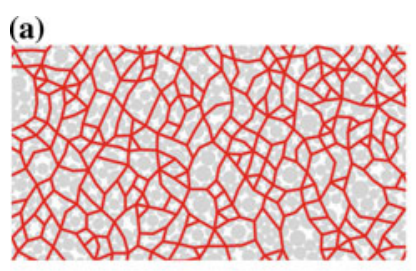

(b)

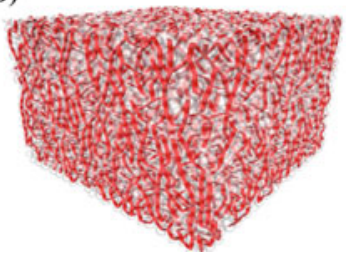

Fig. 1 Contact networks in 2D and 3D granular packings

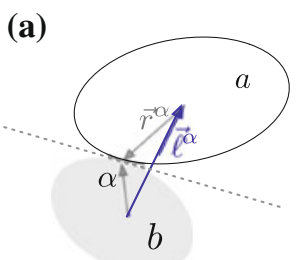

(b)

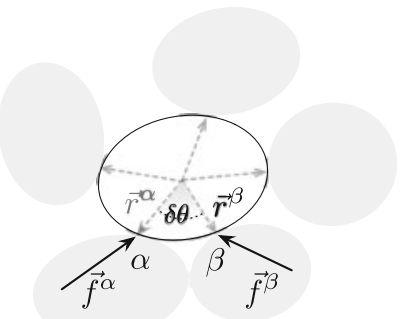

Fig. 2 a Contact geometry; b A particle environment with angular exclusions

perse systems where $P_{\ell n}(\ell, \boldsymbol{n}) \approx P_{\ell}(\ell) P_{n}(\boldsymbol{n})$ and the distribution of branch length $P_{\ell}$ is narrow. Thus the orientation part $P_{n}(\boldsymbol{n})$ contains most of the information.

Yet, $P_{n}$ is too rich to be accessed easily from experiments or handled theoretically. It is proposed to perform a moment expansion of this quantity and retain only the lowest-order terms. The $\pi$-periodicity implies $\langle\boldsymbol{n}\rangle=0$ and therefore the lowest-order moment is the well-known fabric tensor defined by [7] $\boldsymbol{F}=\langle\boldsymbol{n} \otimes \boldsymbol{n}\rangle \equiv \int_{\Omega} \boldsymbol{n} \otimes \boldsymbol{n} P_{n}(\boldsymbol{n}) d \boldsymbol{n}$, where $\otimes$ denotes dyadic product. Since $\boldsymbol{n} \cdot \boldsymbol{n}=1$, we have $\operatorname{tr}(\boldsymbol{F})=1$. Hence, the coordination number $z$ and the fabric tensor can be combined in a single enriched tensor defined by

$G_{i j}=\frac{z}{2}\left\langle n_{i} n_{j}\right\rangle \equiv \frac{z}{2} \int_{\Omega} n_{i} n_{j} P_{n}(\boldsymbol{n}) d \boldsymbol{n}$,

where the roman subscripts denote vector components. Note that $\boldsymbol{G}$ is nothing but the fabric tensor per particle while $\boldsymbol{F}$ is per contact.

The tensor $\boldsymbol{G}$ may easily be evaluated from discrete simulation data by $G_{i j}=\left(\sum_{\alpha} n_{i}^{\alpha} n_{j}^{\alpha}\right) / N_{p}$, where $\boldsymbol{n}^{\alpha}$ is the unit branch vector of contact $\alpha$ and $N_{p}$ is the total number of particles. In 2D, $\boldsymbol{n}$ is parameterized by its orientation $\theta$ with respect to a fixed frame and we have $\boldsymbol{n}=(\cos \theta, \sin \theta)$. In $3 \mathrm{D}$, using spherical coordinates with azimuth and zenith angles $\theta$ and $\phi$, we have $\boldsymbol{n}=(\sin \theta \cos \phi, \sin \theta \sin \phi, \cos \theta)$. For convenience, we assume here axial symmetry around the zenith axis so that $\boldsymbol{G}$ and $P_{n}$ are independent of $\phi$. Let $\left\{G_{i}\right\}$ be the principal values and $\theta=\theta_{c}=0$ the major principal direction. We define the following invariants of $\boldsymbol{G}$ in $2 \mathrm{D}$ :

$p_{G} \equiv \frac{G_{1}+G_{2}}{2}=\frac{z}{4}$
$q_{G} \equiv \frac{G_{1}-G_{2}}{2}=\frac{z}{4}\langle\cos 2 \theta\rangle$

and in 3D:

$p_{G} \equiv \frac{G_{1}+G_{2}+G_{3}}{3}=\frac{z}{6}$

$q_{G} \equiv \frac{G_{1}-G_{3}}{3}=\frac{z}{12}\left\langle 3 \cos ^{2} \theta-1\right\rangle$

By symmetry, we have $G_{2}=G_{3}$. We note that $3 \cos ^{2} \theta-1$ is the spherical harmonics function usually denoted $Y_{2}^{0}$, which together with $Y_{0}^{0}=1$ are the only second-order basis func- 
tions compatible with axial symmetry. The spherical harmonics expansion is the natural 3D counterpart of the Fourier expansion in $2 \mathrm{D}$ which is easily read in the above definitions. The average $\langle\cos 2 \theta\rangle$ or $\left\langle 3 \cos ^{2} \theta-1\right\rangle$ represents the anisotropy and it may be evaluated either from $P_{n}$ (see below) or directly from the numerical data.

Given its invariants, the enriched fabric tensor $\boldsymbol{G}$ may be represented by a Mohr circle in the space of its normal and tangential projections $G_{n}(\boldsymbol{m})=\boldsymbol{G} \boldsymbol{m} \cdot \boldsymbol{m}$ and $G_{t}(\boldsymbol{m})=\boldsymbol{G} \boldsymbol{m}$. $\boldsymbol{t}$, respectively, along arbitrary space direction $\boldsymbol{m}$ parameterized by the angle $\theta$. Simple algebra yields

$$
\begin{aligned}
G_{n}(\theta) & =p_{G}+q_{G} \cos 2 \theta, \\
G_{t}(\theta) & =q_{G} \sin 2 \theta,
\end{aligned}
$$

in $2 \mathrm{D}$ and

$$
\begin{aligned}
G_{n}(\theta) & =p_{G}+q_{G}\left\{3 \cos ^{2} \theta-1\right\}, \\
G_{t}(\theta) & =\frac{3}{2} q_{G} \sin 2 \theta,
\end{aligned}
$$

in $3 \mathrm{D}$.

The representation of the fabric by a second-order tensor through Eq. (1) keeps only the lowest mode of the Fourier (2D) or spherical harmonics (3D) spectrum of $P_{n}$ [5]:

in $2 \mathrm{D}: P_{n}(\theta)=\frac{1}{\pi}\{1+a \cos 2 \theta+\cdots\}$,

in 3D: $P_{n}(\theta)=\frac{1}{2 \pi}\left\{1+a\left(3 \cos ^{2} \theta-1\right)+\cdots\right\}$,

where $a$ is the leading fabric anisotropy. Equation (1) with the expansions (10) and (11) lead to the following relations:

in 2D: $a=2\langle\cos 2 \theta\rangle$,

in $3 \mathrm{D}: \quad a=\frac{5}{4}\left\langle 3 \cos ^{2} \theta-1\right\rangle$.

With this expression of the anisotropy, we see that the description of the fabric by $\boldsymbol{G}$ is equivalent to a description by the truncated expansions:

$$
\begin{aligned}
& \text { in 2D: } \quad E(\theta)=\frac{z}{2} P_{n}(\theta) \approx \frac{z}{2 \pi}\{1+a \cos 2 \theta\}, \\
& \text { in 3D: } E(\theta)=\frac{z}{2} P_{n}(\theta) \approx \frac{z}{4 \pi}\left\{1+a\left(3 \cos ^{2} \theta-1\right)\right\} .
\end{aligned}
$$

The function $E(\theta)$ may assume various shapes with several modes of unequal amplitudes depending on the history or preparation method. Most of time, however, such configurations are erased as a result of shearing and the subsequent shear histories do not induce modes of order higher than two, so that Eqs. (14) and (15) offer a fair approximation of $E(\theta)$. Figure 3 shows two examples of $E$ in the two samples of Fig. 1 before and after shearing. The distribution is fitted by a second-order harmonic approximation, and higher-order terms may be neglected. In this case, the angular dependence of $G_{n}(\theta)$ coincides with that of $E$.
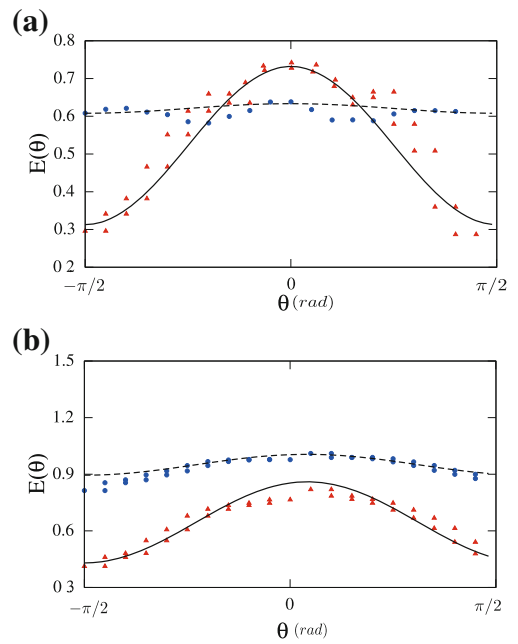

Fig. 3 Distribution of branch-vector orientations in 2D a and 3D b before (circles) and after (triangles) shearing with major principal strain-rate direction along $\theta=0$. The solid lines are harmonic fits

In this way, the fabric state at leading order is represented by $\boldsymbol{G}$ or $E(\theta)$, both characterized by the three variables $z, a$ and $\theta_{c}$. The coordination number $z$ is a simple scalar, but the fabric anisotropy $a$ is associated with the major principal direction $\theta_{c}$ of $\boldsymbol{G}$. When the evolution of the fabric is studied, it is convenient to consider the "signed" anisotropy $a^{\prime} \equiv a \cos 2\left(\theta_{0}-\theta_{c}\right)$ in $2 \mathrm{D}$ or $a^{\prime} \equiv a\left\{3 \cos ^{2}\left(\theta_{0}-\theta_{c}\right)-1\right\}$ in $3 \mathrm{D}$, where $\theta_{0}$ is a fixed space direction, which may be the initial value of $\theta_{c}$.

\section{Evolution with shear strain}

The fabric evolution is governed by three elementary "events": (1) gain of contact, (2) loss of contact and (3) rotation of branch vectors. The gain and loss events are discontinuous whereas the rotation of branch vectors is a continuous advection of enduring contacts in the angular interval $[0, \pi]$. Let $\dot{\boldsymbol{\varepsilon}}$ be the strain rate tensor. These events occur at different rates depending on the space direction $\theta$ : a gain rate $I^{+}(\dot{\varepsilon}, \theta)$, a loss rate $I^{-}(\dot{\boldsymbol{\varepsilon}}, \theta)$ and a current $J(\dot{\boldsymbol{\varepsilon}}, \theta)$ of advected contacts, respectively. The evolution of $E$ is thus governed by a detailed balance equation:

$\frac{\partial E}{\partial t}+\frac{\partial J}{\partial \theta}=I=I^{+}-I^{-}$

We expect that $I^{+}$has its largest value along the direction of contraction whereas $I^{-}$is maximal along the direction of extension. The current $J$ is basically given by the number density of contacts times the angular velocity in each direction. The three functions $I^{+}, I^{-}$and $J$ depend on the velocity field in the vicinity of the particles. An example of the averaged velocity field is shown in Fig. 4. Without entering the 


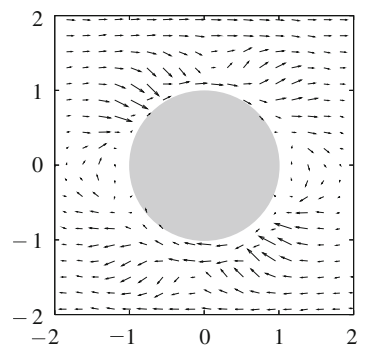

Fig. 4 The field of relative velocities from numerical simulations averaged in the vicinity of all particles during simple shear deformation of a packing of disks along the horizontal direction. The distances are normalized by the average branch-vector length $\langle\ell\rangle$, which defines the radius of the mutual exclusion distance between particles represented by the gray circle in the center

details here, we observe that the far field (uniform velocity gradient) is modified in the vicinity of the particles within a distance of the order of the average branch-vector length. However, this velocity field varies in proportion to the macroscopic velocity gradient although with dependencies on the fabric tensor itself.

Note that in the absence of $J$, the relative number $E$ of contacts in each direction would simply be an accumulation of the contacts induced by loss and gain and a steady state would never be reached. However, in the steady state we have $\partial J / \partial \theta=I$, which means that, the contacts are gained on the average in the direction of contraction and advected towards the direction of extension where they are lost. In this way, the memory of the initial configuration is erased by shear deformation and the critical state may be described as a steady shear state in which, in spite of steric exclusions, the fabric reflects the average strain-rate tensor and may thus be described to a good approximation by a second-order fabric tensor or unimodal distributions of contact orientations.

Figure 5 shows the evolution of the fabric parameters $z$ and $a^{\prime}$ during shear as a function of the cumulative shear strain $\varepsilon_{q}=\varepsilon_{1}-\varepsilon_{2}$, where $\varepsilon_{1}$ and $\varepsilon_{2}$ are the principal values of the strain tensor, for an initially dense system $z_{0}=z\left(\varepsilon_{q}=0\right)=4$ and an initially loose system $z_{0}=3.3$, as well as in the case where the shear deformation is reversed in the steady state. Notice that the terms "dense" and "loose" will refer throughout this paper to the connectivity of the contact network rather than its packing fraction. The numerical simulations were performed by means of the contact dynamics method [2]. The samples are composed of 5,000 disks of weak size polydispersity prepared by isotropic compaction so that $a_{0}^{\prime}=a^{\prime}\left(\varepsilon_{q}=\right.$ $0)=0$. During shear, the average stress $p$ is kept constant and a constant shear rate $\dot{\varepsilon}_{q}$ is applied. We observe an initial rapid decrease of $z$ in the dense case as a consequence of the quasi-instantaneous loss of contacts in the direction of extension. This leads at the same time to an increasingly larger structural anisotropy $a^{\prime}$ with an excess of contacts oriented along
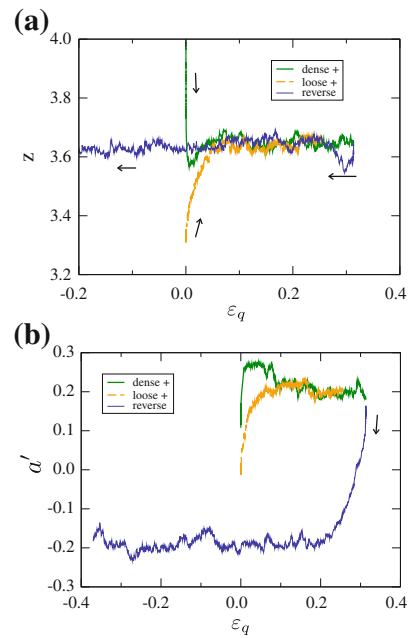

Fig. 5 The evolution of fabric parameters, coordination number (a) and "signed" anisotropy (b). Different curves represent different initial values of the packing fraction

the major principal strain-rate direction $\theta_{\varepsilon}$. A similar mechanism is at work in the loose system with a gain of contacts along $\theta_{\varepsilon}$ and an increase of $a^{\prime}$. After a transient $\varepsilon_{q} \simeq 0.1$, a steady state with $z^{*} \simeq 3.65$ and $a^{*} \simeq 0.2$ is reached in both samples. When a reverse strain is applied in the steady state, $a^{\prime}$ falls off to zero but then increases in absolute value to reach $a^{*}$ in the new shear direction. As for $z$, its steady-state value $z^{*}$ is reached in the new shear-rate direction after a transient where $z$ first declines as soon as the shear strain is reversed and then increases again towards $z^{*}$. A similar unmonotonic evolution is observed when starting from the dense isotropic state: $z$ first declines to a low value, then increases towards $z^{*}$.

\section{Accessible fabric states}

As a result of local constraints, the fabric parameters do not take arbitrary values. The accessible range of state parameters contains part of the underlying constraints and it may thus partially replace the missing information when a low-order description is used. To illustrate the effect of constraints, let us consider the truncated Fourier function of $E(\theta)$ in 2D and apply it to the contact neighbors of a typical particle. The angular exclusions imply that there can be at most one particle in the angular interval $[-\delta \theta / 2, \delta \theta / 2]$ (see Fig. 2b) [8]:

$\int_{-\frac{\delta \theta}{2}}^{\frac{\delta \theta}{2}} E(\theta) d \theta \leq 1$

This leads to an upper bound $a^{\max }$ on the anisotropy:

$a \leq a^{\max }=\frac{2 \pi}{\sin (\delta \theta)}\left\{\frac{1}{z}-\frac{1}{z^{\max }}\right\}$, 
where $z^{\max }=2 \pi / \delta \theta$ is the upper bound on the coordination number due to angular exclusions. However, the function $E(\theta)$ can not be used to describe the local environments because of steric exclusions. We introduce here a simple model based on the fabric tensor $\boldsymbol{G}$ in order to account, though in a less strict way, for the local constraints.

In a co-rotating reference frame with axes oriented along $\theta_{c}\left(\varepsilon_{q}\right)$ and $\theta_{c}\left(\varepsilon_{q}\right)+\pi / 2$, the fabric state is defined by its position in the space of coordinates $z$ and $a$. We assume that $z$ is bounded between two limits $z^{\min }$ and $z^{\max }$. The upper bound $z^{\max }$ is dictated by steric exclusions as discussed above. The lower bound $z^{\text {min }}$ reflects the condition of mechanical equilibrium. For example, stable particles often involve more than three contacts in 2D and more than four contacts in $3 \mathrm{D}$. We first consider exclusively the $2 \mathrm{D}$ case. The results for the $3 \mathrm{D}$ system will be given at the end of this section.

We define two limit states: (1) the loosest isotropic state, characterized by $G_{t}=0$ and $G_{n}^{\min }=z^{\min } / 4$, and (2) the densest isotropic state, characterized by $G_{t}=0$ and $G_{n}^{\max }=$ $z^{\max } / 4$. All accessible fabric states, represented by a Mohr circle on the $\left(G_{n}, G_{t}\right)$ plane as shown in Fig. 6, are enclosed between these two limit states, so that $G_{1} \leq G_{n}^{\max }$ and $G_{2} \geq$ $G_{n}^{\text {min }}$. Within this range $a$ and $z$, which describe the current geometrical state, are independent parameters unless one of the two equalities $G_{2}=G_{n}^{\min }$ or $G_{1}=G_{n}^{\max }$ occurs, in which case $p_{G}$ and $q_{G}$ may no more evolve independently. The condition $G_{2}=G_{n}^{\min }$ implies $p_{G}-q_{G}=z_{\min } / 4$, which together with Eqs. (3) and (12) yields $a=2\left(1-z^{\min } / z\right)$. This limit corresponds to a loss saturation limit where the mechanical equilibrium implies that no contacts may be lost along the direction of extension. In the same way, the condition $G_{1}=G_{n}^{\max }$ implies $p_{G}+q_{G}=z_{\min } / 4$, which together with Eqs. (3) and (12) yields $a=2\left(z^{\max } / z-1\right)$. This is the gain saturation limit where no contacts may be gained along the direction of contraction. Hence, all accessible fabric states in the space $(z, a)$ belong to a domain defined by

$a^{\max }(z)=2 \min \left\{1-\frac{z^{\min }}{z}, \frac{z^{\max }}{z}-1\right\}$.

The largest anisotropy $a^{\star}$ occurs when both conditions $G_{1}=G_{n}^{\max }$ and $G_{2}=G_{n}^{\min }$ are satisfied simultaneously. Hence,

$a^{\star}=a^{\max }\left(z^{\star}\right)=2 \frac{z^{\max }-z^{\min }}{z^{\max }+z^{\min }}$ with $z^{\star}=\frac{1}{2}\left(z^{\min }+z^{\max }\right)$.

This state corresponds to a Mohr circle touching the line $G_{t}=\tan \alpha G_{n}$ where $\sin \alpha=a^{\star} / 2$, as shown in Fig. 6 b.

Figure 7 shows the joint evolution of $a$ and $z$ in a simulated biaxial compression of two initially isotropic samples with coordination numbers $z_{0}=3.1$ and $z_{0}=3.7$. In both simulations, the fabric tends to the critical state with $z^{\star} \simeq 3.35$ and $a^{\star} \simeq 0.24$ and coinciding with the state of maximum
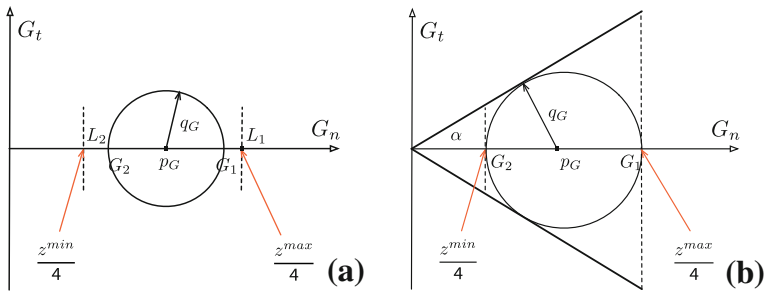

Fig. 6 a Fabric state represented by a Mohr circle. $L_{1}$ and $L_{2}$ are the limit isotropic states. $\mathbf{b}$ The critical state on the fabric plane. The cone represents the limit of maximum anisotropy reached when the Mohr circle touches the limit states in the critical state

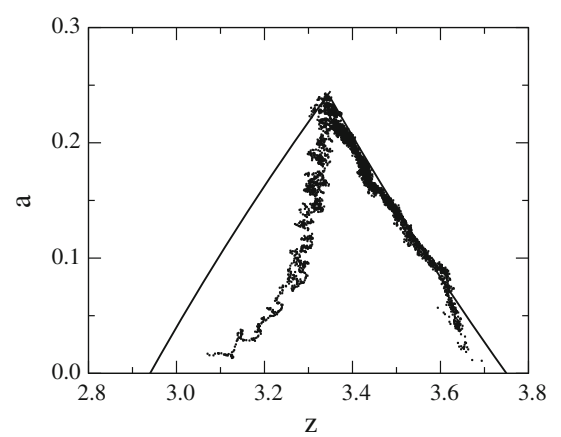

Fig. 7 Fabric evolution in the $(z, a)$ space. The solid lines represent the limits states predicted by Eq. (19)

anisotropy in the model. Remarkably, the anisotropy of the dense packing reaches and then follows closely a limit line given by Eq. (19). The values of $z^{\max }$ and $z^{\min }$ were obtained from the those of $z^{\star}$ and $a^{\star}$ using Eq. (20). In the case of the loose sample, the path remains entirely inside the accessible domain and the boundary is reached only at the critical state.

This description provides a geometrical interpretation of the critical state. The gain and loss saturation lines meet at the critical state. In this sense, the critical state corresponds to a fully saturated state where both contact gain along the direction of contraction and contact loss along the direction of extension are saturated although the shearing continues to induce gain and loss of contacts in different directions. From this viewpoint, the critical state simply reflects the limit isotropic states. The critical-state anisotropy $a^{\star}$ increases with $z^{\max }-z^{\min }$. The shape, size and frictional characteristics of the particles may therefore influence $a^{\star}$ via $z^{\min }$ and $z^{\max }$. Figure 8 shows $a z$ as a function of $z$ for five different simulations with the same sample of 13,000 disks with $z_{0}=3.72$ but different values of $\mu .{ }^{1}$ According to (19), the limit states in this representation are straight lines. The data follow different gain-saturation limit lines with $z^{\max }$ varying between 3.7 and 3.8, and reach a peak state followed by a critical state in which $z^{\star}$ decreases as $\mu$ increases whereas $a^{\star}$ increases nearly as $1 / z^{\star}$. Since $z^{\max }$ varies slightly with $\mu$, the dom-

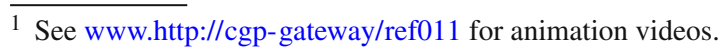




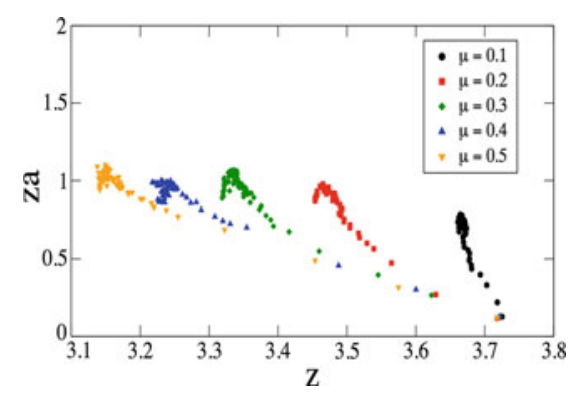

Fig. 8 Fabric evolution in the space $(z, a z)$ for different values of the friction coefficient $\mu$ and the same initial isotropic state. The dashed lines are approximate fits to the reference lines predicted by the model

inant effect of increasing $\mu$ is to allow for lower values of $z^{\mathrm{min}}$ and thus larger values of $a^{\star}$. It is to be noted that a small systematic deviation from the linear law is observed in the final approach to the critical point. Such a small effect may well be due to the truncation of $P_{n}$ to second order, as, at maximum anisotropy, our (angularly) local interpretation of $E$ from its first two moments becomes fragile.

The accessible fabric states may be modeled also in 3D by means of the Mohr circle on the fabric plane. It can be shown that, under axial symmetry, the critical state is characterized by

$$
\begin{aligned}
z^{*} & =\frac{1}{3}\left(z^{\max }+2 z^{\min }\right), \\
a^{*} & =\frac{5}{2} \frac{z^{\max }-z^{\min }}{z^{\max }+2 z^{\min }} .
\end{aligned}
$$

For example, for the typical values $z^{\min }=4$ and $z^{\max }=6$ of a sphere packing, we get $z^{\star} \simeq 4.6$ and $a^{\star} \simeq 0.35$, which are plausible critical state values in 3D. It is obvious that $z$ can take much larger values, but such states are not reachable by shearing. When a packing with a high value of $z$ is sheared, the accessible range of fabric parameters, as defined by the model, may be reached from the outside, but the subsequent evolution is likely to take place only inside this range. Various protocols might be designed for the estimation of $z^{\max }$ and $z^{\text {min }}$ as limit values of the range of accessible fabric states. In this respect, our model may also be regarded as an executive framework in which those values are simply determined from the $(z, a)$ data and interpreted as state-independent parameters characterizing a granular material.

\section{Conclusion}

In this paper, a general framework for fabric evolution was presented with the guiding idea that a physical plastic model of granular materials should be based in the first place on low-order parameters pertaining to the granular microstructure but accounting more or less strictly for steric exclusions and mechanical equilibrium of the particles. We introduced a fabric tensor that, by combining the coordination number and fabric anisotropy, allows for a simple tensorial representation of the fabric states by means of Mohr circles. We also briefly discussed how low-order fabrics are induced by homogeneous shearing, and numerical data of fabric evolution were presented for loose and dense isotropic states and reversed strains from the critical state. Finally, a model was introduced for the range of accessible fabric states. Assuming limit values of the coordination number, reflecting the local constraints, this model predicts in a simple way limit lines and an upper bound on the fabric anisotropy. It provides also a geometrical interpretation of the critical state as a "saturated" state reflecting particle-scale constraints.

\section{References}

1. Azéma, E., Saussine, G., Radjai, F.: Quasistatic rheology, force transmission and fabric properties of a packing of irregular polyhedral particles. Mech. Mat. 41, 729-741 (2009)

2. Radjai, F., Richefeu, V.: Contact dynamics as a nonsmooth discrete element method. Mech. Mat. 41, 715-728 (2009)

3. Radjai, F., Roux, S.: Turbulentlike fluctuations in quasistatic flow of granular media. Phys. Rev. Lett. 89(6), 064302 (2002)

4. Radjai, F., Roux, S.: Contact dynamics study of 2D granular media: critical states and relevant internal variables. In: Hinrichsen, H., Wolf, D.E. (eds.) The Physics of Granular Media, pp. 165-186. Wiley-VCH, Weinheim (2004)

5. Rothenburg, L., Bathurst, R.J.: Analytical study of induced anisotropy in idealized granular materials. Geotechnique 39, 601614 (1989)

6. Roux, S., Radjai, F.: Statistical approach to the mechanical behavior of granular media. In: Aref, H., Philips, J. (eds.) Mechanics for a New Millennium, pp. 181-196. Kluwer Academic Publication, The Netherlands (2001)

7. Satake, M.: Fabric tensor in granular materials. In: Vermeer, P.A., Luger, H.J. (eds.) Proceedings of the IUTAM Symposium on Deformation and Failure of Granular Materials, Delft, pp. 63-68. A.A. Balkema, Amsterdam (1982)

8. Troadec, H., Radjai, F., Roux, S., Charmet, J.: Model for granular texture with steric exclusion. Phys. Rev. E 66(41), 041305-1 (2002)

9. Wood, D.: Soil Behaviour and Critical State Soil Mechanics. Cambridge University Press, Cambridge (1990) 